\title{
Activity Recognition with Moving Cameras and Few Training Examples: Applications for Detection of Autism-Related Headbanging
}

\author{
Peter Washington \\ Stanford University \\ peterwashington@stanford.edu \\ Emilie Leblanc \\ Stanford University \\ eleblanc@stanford.edu \\ Kelley Paskov \\ Stanford University \\ kpaskov@stanford.edu
}

\author{
Aaron Kline \\ Stanford University \\ akline@stanford.edu \\ Cathy Hou \\ Stanford University \\ cathyhou@stanford.edu \\ Brianna Chrisman \\ Stanford University \\ briannac@stanford.edu
}

\author{
Onur Cezmi Mutlu \\ Stanford University \\ cezmi@stanford.edu \\ Nate Stockham \\ Stanford University \\ stockham@stanford.edu \\ Dennis P. Wall \\ Stanford University \\ dpwall@stanford.edu
}

\begin{abstract}
Activity recognition computer vision algorithms can be used to detect the presence of autism-related behaviors, including what are termed "restricted and repetitive behaviors", or stimming, by diagnostic instruments. Examples of stimming include hand flapping, spinning, and head banging. One of the most significant bottlenecks for implementing such classifiers is the lack of sufficiently large training sets of human behavior specific to pediatric developmental delays. The data that do exist are usually recorded with a handheld camera which is itself shaky or even moving, posing a challenge for traditional feature representation approaches for activity detection which capture the camera's motion as a feature. To address these issues, we first document the advantages and limitations of current feature representation techniques for activity recognition when applied to head banging detection. We then propose a feature representation consisting exclusively of head pose keypoints. We create a computer vision classifier for detecting head banging in home videos using a time-distributed convolutional neural network $(\mathrm{CNN})$ in which a single CNN extracts features from each frame in the input sequence, and these extracted features are fed as input to a long short-term memory (LSTM) network. On the binary task of predicting head banging and no head banging within videos from the Self Stimulatory Behaviour Dataset (SSBD), we reach a mean F1-score of $90.77 \%$ using 3-fold cross validation (with individual fold F1-scores of $83.3 \%, 89.0 \%$, and $100.0 \%$ ) when ensuring that no child who appeared in the train set was in the test set for all folds. This work documents a successful process for training a computer vision classifier which can detect a particular human motion pattern with few training examples and even when the camera recording the source clip is unstable. The process of engineering useful feature
\end{abstract}

This work is licensed under a Creative Commons Attribution International 4.0 License.

CHI '21 Extended Abstracts, May 08-13, 2021, Yokohama, Japan

(c) 2021 Copyright held by the owner/author(s).

ACM ISBN 978-1-4503-8095-9/21/05.

https://doi.org/10.1145/3411763.3451701 representations by visually inspecting the representations, as described here, can be a useful practice by designers and developers of interactive systems detecting human motion patterns for use in mobile and ubiquitous interactive systems.

\section{CCS CONCEPTS}

- Human-centered computing; • Computing methodologies $\rightarrow$ Machine learning; Learning paradigms; Supervised learning; Artificial intelligence; Computer vision; Computer vision tasks; Activity recognition and understanding;

\section{KEYWORDS}

Activity recognition, repetitive motions, motion detection, autism, machine learning

ACM Reference Format:

Peter Washington, Aaron Kline, Onur Cezmi Mutlu, Emilie Leblanc, Cathy Hou, Nate Stockham, Kelley Paskov, Brianna Chrisman, and Dennis P. Wall. 2021. Activity Recognition with Moving Cameras and Few Training Examples: Applications for Detection of Autism-Related Headbanging. In CHI Conference on Human Factors in Computing Systems Extended Abstracts (CHI '21 Extended Abstracts), May 08-13, 2021, Yokohama, Japan. ACM, New York, NY, USA, 7 pages. https://doi.org/10.1145/3411763.3451701

\section{INTRODUCTION}

The increasing ubiquity of mobile devices in conjunction with advances in artificial intelligence (AI), and in particular machine learning (ML), are enabling accessibility of healthcare solutions for traditionally underserved populations. While the prevalence of autism exceeds 1 in 60 children [22,40], current estimates are that over $80 \%$ of counties in the United States lack access to autism diagnostics [44]. As a result, families will often need to wait over a year to receive a diagnosis for autism, particularly in rural areas and in lower socioeconomic communities [25]. This prolonged and excessive wait time is particularly problematic for pediatric developmental delays like autism, where earlier diagnosis and resulting behavioral interventions result in more effective clinical outcomes [13-14].

Research has shown that autism features useful for diagnosis and continuous phenotyping can be detected in short home videos 
taken by smartphones [17-19, 23-24, 38, 54-55, 59, 64]. Interactive devices such as smartphones are increasingly being evaluated for use in at-home digital diagnostics and therapies which adapt to the needs of the child [43]. For diagnostics, computer vision classifiers measure the behavior of the child, and statistical measurements are used to provide a diagnosis or screening [13-14, 58, 75]. For digital therapeutics, the classifiers measure the response of the child to an interactive game or task designed to provide behavioral therapy for the child [11-12, 35, 56-57, 65-66].

Unfortunately, autism-related behaviors are currently particularly difficult to classify with out-of-the-box computer vision approaches. Existing activity recognition classifiers have been developed and evaluated on common activities like running, throwing a basketball, and dancing [9]. Restricted and repetitive autism movements (i.e., stimming), such as hand flapping, head banging, and rocking back and forth, have not been the focus of computer vision efforts, largely because of a dearth of training data for such domain specific behaviors. An additional issue is that while many activity recognition algorithms assume a stable and still camera, many home videos of children exhibiting stimming behaviors are recorded from an unstable handheld camera source. There is a need for computer vision methods which can distinguish and detect activities relevant to marginalized populations where massive training sets do not exist and only relatively small amounts of data from potentially unstable camera sources are available.

In this paper, we document the process of designing a feature representation method for head banging detection. We visually inspect several traditional feature representation methods for activity recognition on video clips enriched for head banging recorded with a handheld camera. Through discussing the advantages and disadvantages of each technique with respect to the visual feature representation, we arrive at a pose-based representation of only head keypoints. We train a time-distributed convolutional neural network $(\mathrm{CNN})$ which extracts features that are fed into a long short-term memory (LSTM) network. We train this model to distinguish video clips containing "head banging" from "not headbanging" using a balanced set of videos, resulting in a $90.77 \%$ F1-score during 3-fold cross-validation. This paper provides an example of training a human activity classifier for detection of a specific activity using few data points collected from unstable camera streams. We believe that the simple yet reasoned process outlined in this study will be of utility to engineers of ML-powered interactive systems centered around detecting human behavior.

\section{RELATED WORK}

We discuss examples of both advances in computer vision techniques for detecting stimming behaviors and corresponding interactive systems which target stimming behaviors in children with developmental delays. Because this research area is plentiful, we focus our literature review particularly on autism and pediatrics literature.

\subsection{Activity recognition with moving cameras}

General-purpose activity recognition with moving cameras has been explored in prior work. Most prior approaches are based on representations derived from optical flow, an approach we describe in more detail in Section 3. Wu et al. obtain particle trajectories through optical flow over time and decompose the trajectories into a background and foreground component [71]. The object motion and camera motion are used to predict activity. Rezazadegan et al. identify "action regions" through a multi-step process [49]. They first identify the outline of the body ("boundary mask") using features derived from optical flow. The boundary mask is predicted with structured random forests. To obtain the final region of interest, they employ edge-based object detection methods [74] on the binary boundary mask. They achieve $74.73 \%$ success rate on the UCF101 dataset and $42.1 \%$ success rate on HMDB. Avgerinakis et al. identify background regions displaying motion artefacts using optical flow and superpixels; dense trajectories and motion extracted from these regions are used as features to detect activity, achieving $56.2 \%$ accuracy on the UCF sports dataset [2]. Optical flow-based feature representations maintain artefacts from the human subject's physical appearance, thus requiring large datasets to train a classifier.

Other activity recognition literature use features derived from pose estimation. Pose estimations are ideal for smaller datasets due to their robustness against the physical appearance of the human subject. A stick figure is used to represent the human's body at every step, thereby reducing overfitting. Okumura et al. use hand keypoints to detect cooking-related activities from an egocentric camera [45]. Dousty and Zariffa use hand detection, pose estimation, and arm orientation estimation to detect tenodesis grasp [16]. Pose estimation approaches can be easier to implement than flow-based methods, as they often do not require extensive and complex postprocessing steps.

\subsection{Computer vision detection of stimming}

Computer vision approaches can enable scalable and affordable "behavioral phenotyping" of children with autism [50]. While general purpose activity recognition algorithms can perform well on a broad set of actions, it can be useful to leverage a custom feature representation for more domain-specific tasks. Here, we discuss prior computer vision efforts for automatically identifying motor movements and stereotyped behaviors.

Motor control and movements related to autism include head posture, head movement, and grasping patterns. Researchers have hypothesized that computer vision algorithms can pick up on the generally accepted motor variations between children with autism and neurotypical children. Some studies operate under controlled laboratory conditions. In one such study, Dawson et al. find that toddlers with autism exhibit increased head movement compared to neurotypical controls by tracking facial landmarks [13-14]. In another lab-controlled study, Zunino et al. use a CNN which extracts features which are then fed into an LSTM to detect grasping actions, reaching an accuracy of $72 \%$ for subjects with autism and $77 \%$ for neurotypical subjects [75]. We note that we employed a similar neural network architecture in the present study. Some research efforts have worked towards classification of autism-related motor movements from unstructured video clips. Vyas et al. use a 2D mask R-CNN to distinguish autism from neurotypical behavior in unstructured video clips from a private data set, reporting precision of $72 \%$ and recall of $92 \%$ [58]. 
Advances in computer vision detection require shared and public datasets for training and testing the model. The Self-Stimulatory Behavior Dataset (SSBD) [48] is a dataset of unstructured home videos of children performing one of three self-stimulatory behaviors: headbanging, spinning, and hand flapping. The authors of the dataset build a 3-way classifier distinguishing these 3 behaviors using Space Time Interest Points (STIP) [37] and reach a 50.7\% classification accuracy using 5 -fold cross validation and $47.3 \%$ accuracy using 10-fold cross-validation. We use the SSBD dataset in the present study, as it is the only public dataset, we are aware of containing several labeled examples of self-stimulatory behaviors.

\subsection{Machine learning powered interactive systems for behavioral therapy}

The intersection of HCI and AI for autism has become an area of interest within the HCI community. Interactive systems have been developed to aid in at-home behavioral therapy for children with autism, and these digital therapies are powered by activity recognition models which automatically detect autism-related behaviors. Some works explore how AI can enhance existing interactive technologies for people with autism. Begel et al. leverage emotion detection models for providing feedback about facial expressions during video calling sessions [5]. Bartoli et al. evaluate the potential of games centered around "motion-based" touchless interaction, where sensing devices such as cameras powered by computer vision algorithms track and analyze body movements [4]. On a prototype of this paradigm with a Kinect application, they find that children with autism improved on measures of selective attention and sustained attention after playing the game. Other works explore how AI-powered interactive systems can aid in structured therapy. Mobahi and Karahalios propose that HCI systems can continually monitor the gaze of a child and adapt the treatment accordingly [41]. Robotic therapies powered by computer vision have also demonstrated great promise. Feil-Seifer et al. show that a classifier can determine whether children are attempting to interact with a socially assistive robot in a social manner [21]. Moghadas et al. use computer vision to distinguish children autism from neurotypical children from interaction with a parrot-like robot [42]. A number of HCI works detect autism-related behaviors such as eye contact [72], atypical prosody [6], and emotion expression [29-33, 46, 56-57, 65-66] for use in wearable autism therapies. Continued improvements in the underlying computer vision algorithms powering these interactive systems will enable higher precision interactions in targeted therapeutic games for autism.

\section{ACTIVITY-BASED FEATURE EXTRACTION WITH MOVING CAMERAS}

In the absence of a massive and sufficiently heterogeneous dataset, activity recognition algorithms from video streams require feature extraction of relevant features to avoid overfitting to the training data. Before building our classifier, we explored three common activity recognition methods: Lucas-Kanade optical flow, dense optical flow, and pose estimation via keypoint detection. We use videos contained in the Self-Stimulatory Behavior Dataset (SSBD) [48], a dataset of unstructured home videos of children performing one of three self-stimulatory behaviors: headbanging, spinning, and hand flapping. The dataset is scraped from public domain videos posted on video sharing websites such as YouTube, Vimeo, and Dailymotion. Each of the three behaviors is marked with a timestamp. We manually visualize Lucas-Kanade optical flow, dense optical flow, and pose estimation via keypoint detection for several clips and determined that only a modified version of pose estimation would be feasible with moving cameras. Figure 1 displays the representation of 6 sequential frames using each technique for a video clip exhibiting head banging. We describe the advantages and limits of these feature representation methods below to motivate our eventual approach, and we use Figure 1 as an illustration of problems which occurred consistently across videos.

Dense optical flow computes flow for all points in a frame, resulting in "flow vectors" with a magnitude and direction [20]. Figure 1B displays an example of dense optical flow on a sequence of frames for a video clip of head banging in the SSBD dataset, where direction is encoded as hue and magnitude is encoded as intensity. Clear limitations of dense optical flow representations are immediately clear. For example, movement is detected outside of the region of the child, for example by the pink toy on the table. The child's chair is also sometimes detected as movement. These extra non-relevant movement patterns add much noise to the dense optical flow image, making it a non-ideal representation. One possible solution is to run object detection to crop out the part of the frame without the human, but background artefacts may still remain in this approach. In addition, the flow image appears similar to the outline of the child, making the method prone to overfitting to the child's body shape and camera angle.

Lucas-Kanade optical flow, in contrast to dense optical flow, computes flow for a sparse number of points pre-defined by the user [3], for example detected edges or corners. We create a uniform square grid of tracking points where each point is spaced out by 10 pixels in both the $\mathrm{x}$ and $\mathrm{y}$ direction. Figure $1 \mathrm{C}$ displays the resulting Lucas-Kanade optical flow motion tracking appended to the original image, while Figure 1D displays the tracking in isolation. The Lucas-Kanade method brings its own set of limitations by detecting movement outside of the body to an extent that is more dramatic than dense optical flow. This technique is particularly sensitive to background movement detection associated with slight shifts in the camera.

Pose estimation is a technique which is more robust to camera movement compared to optical flow. We use OpenPose realtime multi-person pose estimation [7-8, 51, 68] to track skeletal keypoints in each frame. OpenPose uses a $\mathrm{CNN}$ which is trained to predict part affinity fields, or flow fields representing relationships between body parts, and confidence maps which encode body part locations. Unlike optical flow, OpenPose predicts each frame independently of the surrounding frames. Figure 1E displays the estimated skeletal pose appended to the original image, while Figure 1D displays the pose skeleton in isolation. There are some clear limitations to using unmodified pose estimation. The noisy skeleton problem is a documented issue which arises when body parts are self-occluded [39, 73]. We observe that body part occlusion is a frequent occurrence in unstructured home videos, making unmodified pose estimation a non-ideal feature representation.

To account for the body part occlusion issue, which would inject unnecessary noise which would confuse the classifier, we modify 

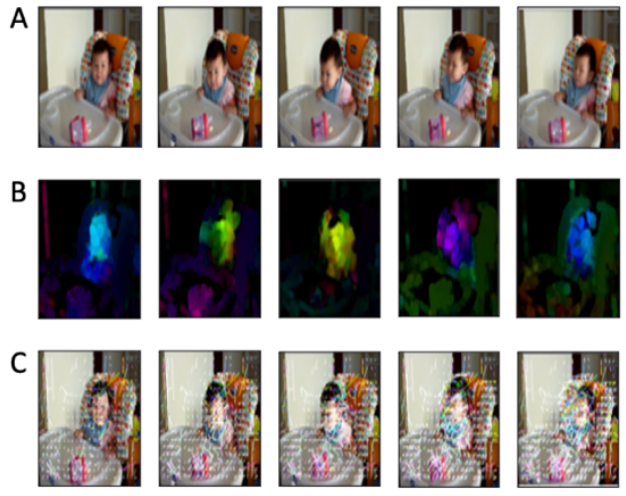
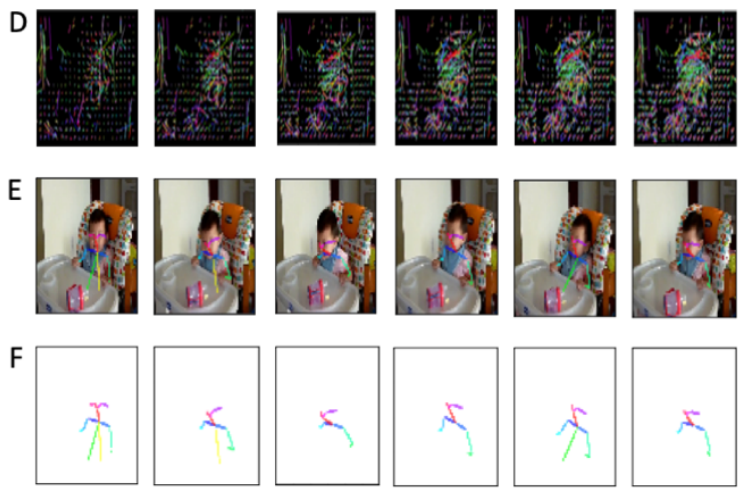

Figure 1: Representation of 6 sequential frames using popular feature representation techniques for activity recognition for a video clip exhibiting head banging. (A) The unmodified sequential frames. (B) Dense optical flow. (C) Lucas-Kanade optical flow displayed on the original image. (D) Lucas-Kanade optical flow in isolation. (E) Pose estimation using body keypoints displayed on the original image. (F) Pose estimation without the original image in the background. All methods displayed here contain noisy features unrelated to headbanging, many of which occur due to camera movement.

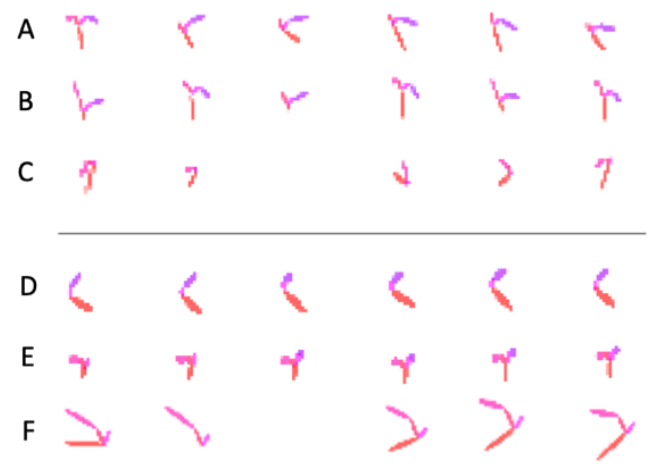

Figure 2: The feature representation used in this paper on headbanging video clips for 3 separate children $(A)-(C)$ and for not head banging video clips for 3 other children (D) - (F). Only head keypoints and lines connecting them are retained in this representation. Every 5 frames in the original video are sampled to generate the input sequence to the neural network ( 7 frames covers one second of time at $\mathbf{3 0}$ frames per second). While head banging clips show differing keypoints for each frame, non-head banging clips show comparatively stable keypoints between frames.

the pose estimation output by only extracting key points in the head region. For a headbanging classifier that assumes the child subject is standing still, we hypothesize that only the head keypoints are necessary for a headbanging classifier. The resulting feature representation is displayed in Figure 2, which contains 3 examples of headbanging sequences (A-C) and 3 examples of non-headbanging sequences (D-F). We qualitatively observe that the headbanging sequences tends to show differing keypoint positions for each frame, while non-head banging clips show comparatively stable keypoints between frames (Figure 2).

\section{TRAINING AND TESTING A HEAD BANGING DETECTOR}

We implement a time-distributed convolutional neural network (CNN) using the Keras [10] Python library with a Tensorflow [1] backend. The time-distributed $\mathrm{CNN}$ is a standard $\mathrm{CNN}$ architecture which learns to extract visual features from each frame in the image sequence. The activated feature maps from each image in the video clip are fed into the corresponding sequence input position in a long short-term memory (LSTM) [28] neural network. We note that the same $\mathrm{CNN}$ architecture and weights are used at each time step in the LSTM rather than a separate CNN trained for each position in the sequence. Figure 3 provides a visualization of the time-distributed CNN architecture. We train using Adam optimization [34] with an initial learning rate of 0.0001 .

We perform 3-fold cross validation, ensuring that no child who appeared in the train set would appear in the test set for all folds. Three folds was the right amount to ensure that a variety of children would appear in both the training and testing sets while still maintaining the ability to evaluate the classifier under multiple partitions of the dataset. In total, we use 27 video clips containing headbanging video clips containing "normal" head motions. To minimize overfitting and increase generalization, we apply the following data augmentations to each frame: rotation at a random interval between -45 and 45 degrees and zooming in with a random zoom factor between 1.0 and 2.0. Although the dataset is small, we believe that the combination of the reduced feature space, data augmentation, and complete separation of children between training and testing sets provides strong validation of the presented technique for detecting head banging.

The mean F1-score, a popular machine learning metric which is the harmonic mean of precision and recall, is $90.77 \%$ across the 3 cross-validation folds. The individual F1-scores per fold are $83.3 \%$, $89.0 \%$, and $100.0 \%$. 


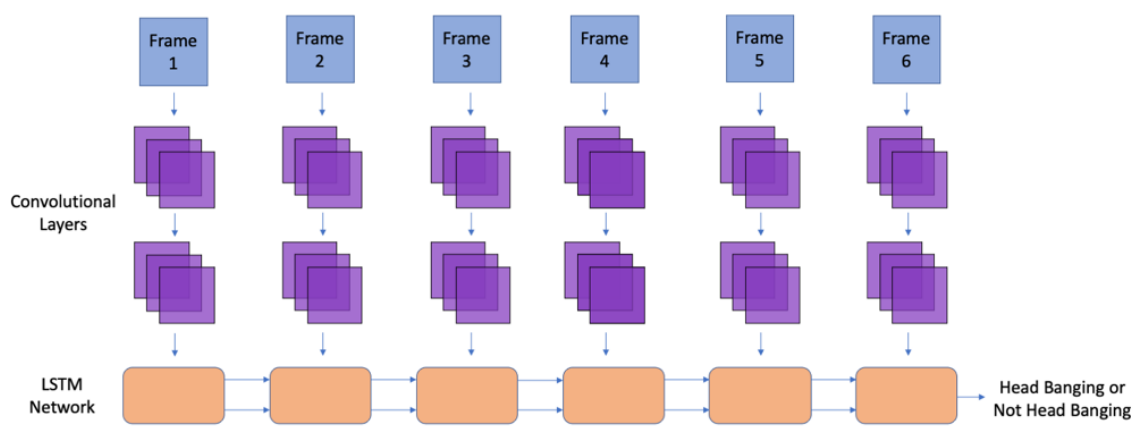

Figure 3: Model architecture for the time-distributed convolutional neural network (CNN) trained to detect hand flapping from short video sequences. Each input frame is fed into a $\mathrm{CNN}$ which extracts visual features from the frame. These features are used as input to an LSTM network which makes the final binary prediction of "head banging" vs. "not head banging".

\section{DISCUSSION, LIMITATIONS, AND FUTURE WORK}

We present a computer vision classifier which can detect head banging in videos recorded with a handheld device. We achieve a $90.77 \%$ F1-score on a subset of video clips from the SSBD dataset. We separate videos by children into the train and test sets for each fold of 3-fold cross validation, ensuring that no child in a video clip which appeared in the train set also appeared in the test set. However, we must admit that head banging classification is an incredibly "easy" computer vision task after extracting the correct feature representation of exclusively head poses, as visualized in Figure 3. This highlights the importance of selecting a robust and representative feature representation when conducting activity-based activity recognition. Other autism-related stimming behaviors, such as hand flapping and body spinning, will likely require further engineering of the feature representation.

There are several limitations to the current pilot study which must be addressed in an expanded and more comprehensive evaluation. While intuition suggests that the reduced noise of the posebased feature representations will result in less overfitting to the human subject's physical appearance and background environment, a formal comparison against optical flow approaches will be required to quantify the actual performance gains. Crucially, we evaluate against a subset of an already small dataset. While it is interesting that the classifier was able to distinguish headbanging from not headbanging with such a small sample, a much larger dataset is required to see how these results generalize. Nevertheless, we believe the preliminary results presented here show that it is possible to detect headbanging with few training samples, and we welcome further work which evaluates the method more comprehensively and with respect to alternative methods.

A major direction for future work will be to evaluate the utility of the head banging model in clinical settings. Remote AI-powered video-based diagnostics and screeners [17-19, 27, 36, 38, 54-55, 5964] as well as telemedical digital therapies [11-12, 15, 26, 47, 5253, 56-57, 65-66, 69-70] are increasingly being developed for autism in children. The efficacy of remote and automated telemedicine is limited to the performance of the underlying AI. Due to the complexity of computer vision for human behavior in general, further innovations and creativity in feature extraction and representation methods are vital to account for noisy datasets and to enable distinguishing of complex human behaviors. Crucially, at-home therapy requires more robust vision models which can account for moving or unsteady cameras, which are conditions that are not usually accounted for by optical flow and other feature representations for activity recognition. Extracting a subset of poses is a good start, but there are potentially more effective and robust representations waiting to be discovered.

There are several HCI considerations which warrant future study. It would be useful to know whether crowd workers can provide reliable activity annotations with transformed images to enables privacy-aware crowdsourcing. For example, while optical flow may not be an effective representation for training ML models, it may preserve the privacy of the clips while still revealing enough information to allow crowd workers to provide correct labels [67]. It will also be important to evaluate how new behavioral classifiers can be integrated into interactive systems, enabling increasingly adaptive user interfaces.

\section{CONCLUSION}

We present a methodology of utility to interaction designers and engineers of interactive systems which measure human motion patterns. We consider the reality that human activity is often tracked with moving cameras, posing an issue for several traditional feature representations such as optical flow or full body keypoints. While we validate our method for head banging detection, a problem that is especially crucial for digital autism diagnostics and adaptive therapeutics, the presented technique can be applied by practitioners of all interactive and AI-powered systems that require measurement of human motion under unstable camera conditions. 


\section{ACKNOWLEDGMENTS}

This work was supported in part by funds to DPW from the National Institutes of Health (1R01EB025025-01, 1R21HD091500-01, 1R01LM013083), the National Science Foundation (Award 2014232), The Hartwell Foundation, Bill and Melinda Gates Foundation, Coulter Foundation, Lucile Packard Foundation, the Weston Havens Foundation, and program grants from Stanford's Human Centered Artificial Intelligence Program, Stanford's Precision Health and Integrated Diagnostics Center (PHIND), Stanford's Beckman Center, Stanford's Bio-X Center, Predictives and Diagnostics Accelerator (SPADA) Spectrum, Stanford's Spark Program in Translational Research, and from Stanford's Wu Tsai Neurosciences Institute's Neuroscience: Translate Program. We also acknowledge generous support from David Orr, Imma Calvo, Bobby Dekesyer and Peter Sullivan. P.W. would like to acknowledge support from Mr. Schroeder and the Stanford Interdisciplinary Graduate Fellowship (SIGF) as the Schroeder Family Goldman Sachs Graduate Fellow.

\section{REFERENCES}

[1] Abadi, Martín, Paul Barham, Jianmin Chen, Zhifeng Chen, Andy Davis, Jeffrey Dean, Matthieu Devin et al. "Tensorflow: A system for large-scale machine learning." In 12th \{USENIX\} symposium on operating systems design and implementation (\{OSDI\} 16), pp. 265-283. 2016.

[2] Avgerinakis, Konstantinos, Katerina Adam, Alexia Briassouli, and Yiannis Kompatsiaris. "Moving camera human activity localization and recognition with motionplanes and multiple homographies." In 2015 IEEE International Conference on Image Processing (ICIP), pp. 2085-2089. IEEE, 2015.

[3] Baker, Simon, and Iain Matthews. "Lucas-kanade 20 years on: A unifying framework." International journal of computer vision 56, no. 3 (2004): 221-255.

[4] Bartoli, Laura, Clara Corradi, Franca Garzotto, and Matteo Valoriani. "Exploring motion-based touchless games for autistic children's learning." In Proceedings of the 12th international conference on interaction design and children, pp. 102-111. 2013

[5] Begel, Andrew, John Tang, Sean Andrist, Michael Barnett, Tony Carbary, Piali Choudhury, Edward Cutrell et al. "Lessons Learned in Designing AI for Autistic Adults." In The 22nd International ACM SIGACCESS Conference on Computers and Accessibility, pp. 1-6. 2020.

[6] Boyd, LouAnne E., Alejandro Rangel, Helen Tomimbang, Andrea Conejo-Toledo, Kanika Patel, Monica Tentori, and Gillian R. Hayes. "SayWAT: Augmenting faceto-face conversations for adults with autism." In Proceedings of the $2016 \mathrm{CHI}$ Conference on Human Factors in Computing Systems, pp. 4872-4883. 2016.

[7] Cao, Zhe, Gines Hidalgo, Tomas Simon, Shih-En Wei, and Yaser Sheikh. "OpenPose: realtime multi-person 2D pose estimation using Part Affinity Fields." IEEE transactions on pattern analysis and machine intelligence 43, no. 1 (2019): 172-186.

[8] Cao, Zhe, Tomas Simon, Shih-En Wei, and Yaser Sheikh. "Realtime multi-person $2 \mathrm{~d}$ pose estimation using part affinity fields." In Proceedings of the IEEE conference on computer vision and pattern recognition, pp. 7291-7299. 2017.

[9] Caba Heilbron, Fabian, Victor Escorcia, Bernard Ghanem, and Juan Carlos Niebles. "Activitynet: A large-scale video benchmark for human activity understanding." In Proceedings of the ieee conference on computer vision and pattern recognition, pp. 961-970. 2015.

[10] Chollet, François. "Keras: The python deep learning library." ascl (2018): ascl-1806.

[11] Daniels, Jena, Jessey N. Schwartz, Catalin Voss, Nick Haber, Azar Fazel, Aaron Kline, Peter Washington, Carl Feinstein, Terry Winograd, and Dennis P. Wall. "Exploratory study examining the at-home feasibility of a wearable tool for social-affective learning in children with autism." NPf digital medicine 1, no. 1 (2018): 1-10.

[12] Daniels, Jena, Nick Haber, Catalin Voss, Jessey Schwartz, Serena Tamura, Azar Fazel, Aaron Kline et al. "Feasibility testing of a wearable behavioral aid for social learning in children with autism." Applied clinical informatics 9, no. 1 (2018): 129

[13] Dawson, Geraldine, Kathleen Campbell, Jordan Hashemi, Steven J. Lippmann Valerie Smith, Kimberly Carpenter, Helen Egger et al. "Atypical postural control can be detected via computer vision analysis in toddlers with autism spectrum disorder." Scientific reports 8, no. 1 (2018): 1-7.

[14] Dawson, Geraldine, and Guillermo Sapiro. "Potential for digital behavioral measurement tools to transform the detection and diagnosis of autism spectrum disorder." JAMA pediatrics 173, no. 4 (2019): 305-306.

[15] Deriso, David, Joshua Susskind, Lauren Krieger, and Marian Bartlett. "Emotion mirror: a novel intervention for autism based on real-time expression recognition." In European Conference on Computer Vision, pp. 671-674. Springer, Berlin, Heidelberg, 2012.
[16] Dousty, Mehdy, and José Zariffa. "Tenodesis Grasp Detection in Egocentric Video." IEEE Journal of Biomedical and Health Informatics (2020).

[17] Duda, Marlena, Jena Daniels, and Dennis P. Wall. "Clinical evaluation of a novel and mobile autism risk assessment." fournal of autism and developmental disorders 46, no. 6 (2016): 1953-1961.

[18] Duda, M., N. Haber, J. Daniels, and D. P. Wall. "Crowdsourced validation of a machine-learning classification system for autism and ADHD." Translational psychiatry 7, no. 5 (2017): e1133-e1133.

[19] Duda, M., R. Ma, N. Haber, and D. P. Wall. "Use of machine learning for behavioral distinction of autism and ADHD." Translational psychiatry 6, no. 2 (2016): e732e732.

[20] Farnebäck, Gunnar. "Two-frame motion estimation based on polynomial expansion." In Scandinavian conference on Image analysis, pp. 363-370. Springer, Berlin, Heidelberg, 2003.

[21] Feil-Seifer, David, and Maja Matarić. "Using proxemics to evaluate human-robot interaction." In 2010 5th ACM/IEEE International Conference on Human-Robot Interaction (HRI), pp. 143-144. IEEE, 2010.

[22] Fombonne, Eric. "The rising prevalence of autism." fournal of Child Psychology and Psychiatry 59, no. 7 (2018): 717-720.

[23] Fusaro, Vincent A., Jena Daniels, Marlena Duda, Todd F. DeLuca, Olivia D'Angelo, Jenna Tamburello, James Maniscalco, and Dennis P. Wall. "The potential of accelerating early detection of autism through content analysis of YouTube videos." PLOS one 9, no. 4 (2014): e93533.

[24] Fusaro, Vincent A., Prasad Patil, Erik Gafni, Dennis P. Wall, and Peter J. Tonellato. "Biomedical cloud computing with amazon web services." PLoS Comput Biol 7, no. 8 (2011): e1002147.

[25] Gordon-Lipkin, Eliza, Jessica Foster, and Georgina Peacock. "Whittling down the wait time: exploring models to minimize the delay from initial concern to diagnosis and treatment of autism spectrum disorder." Pediatric Clinics 63, no. 5 (2016): 851-859.

[26] Haber, Nick, Catalin Voss, and Dennis Wall. "Making emotions transparent: Google Glass helps autistic kids understand facial expressions through augmented-reaiity therapy." IEEE Spectrum 57, no. 4 (2020): 46-52.

[27] Halim, Abbas, Garberson Ford, Stuart Liu-Mayo, Eric Glover, and Dennis P. Wall. "Multi-modular AI Approach to Streamline Autism Diagnosis in Young Children." Scientific Reports (Nature Publisher Group) 10, no. 1 (2020).

[28] Hochreiter, Sepp, and Jürgen Schmidhuber. "Long short-term memory. " Neural computation 9, no. 8 (1997): 1735-1780.

[29] Kalantarian, Haik, Khaled Jedoui, Kaitlyn Dunlap, Jessey Schwartz, Peter Washington, Arman Husic, Qandeel Tariq, Michael Ning, Aaron Kline, and Dennis Paul Wall. "The performance of emotion classifiers for children with parent-reported autism: quantitative feasibility study." FMIR mental health 7, no. 4 (2020): e13174.

[30] Kalantarian, Haik, Khaled Jedoui, Peter Washington, Qandeel Tariq, Kaiti Dunlap, Jessey Schwartz, and Dennis P. Wall. "Labeling images with facial emotion and the potential for pediatric healthcare." Artificial intelligence in medicine 98 (2019): 77-86.

[31] Kalantarian, Haik, Khaled Jedoui, Peter Washington, and Dennis P. Wall. "A mobile game for automatic emotion-labeling of images." IEEE transactions on games 12 , no. 2 (2018): 213-218.

[32] Kalantarian, Haik, Peter Washington, Jessey Schwartz, Jena Daniels, Nick Haber, and Dennis P. Wall. "Guess what?." Journal of healthcare informatics research 3, no. 1 (2019): 43-66

[33] Kalantarian, Haik, Peter Washington, Jessey Schwartz, Jena Daniels, Nick Haber, and Dennis Wall. "A gamified mobile system for crowdsourcing video for autism research." In 2018 IEEE international conference on healthcare informatics (ICHI), pp. 350-352. IEEE, 2018.

[34] Kingma, Diederik P., and Jimmy Ba. "Adam: A method for stochastic optimization." arXiv preprint arXiv:1412.6980 (2014).

[35] Kline, Aaron, Catalin Voss, Peter Washington, Nick Haber, Hessey Schwartz, Qandeel Tariq, Terry Winograd, Carl Feinstein, and Dennis P. Wall. "Superpower glass." GetMobile: Mobile Computing and Communications 23, no. 2 (2019): 35-38.

[36] Kosmicki, J. A., V. Sochat, M. Duda, and D. P. Wall. "Searching for a minimal set of behaviors for autism detection through feature selection-based machine learning." Translational psychiatry 5, no. 2 (2015): e514-e514.

[37] Laptev, Ivan. "On space-time interest points." International journal of computer vision 64 , no. 2-3 (2005): 107-123

[38] Leblanc, Emilie, Peter Washington, Maya Varma, Kaitlyn Dunlap, Yordan Penev, Aaron Kline, and Dennis P. Wall. "Feature replacement methods enable reliable home video analysis for machine learning detection of autism." Scientific reports 10, no. 1 (2020): 1-11.

[39] Li, Wanqing, Zhengyou Zhang, and Zicheng Liu. "Action recognition based on a bag of 3d points." In 2010 IEEE Computer Society Conference on Computer Vision and Pattern Recognition-Workshops, pp. 9-14. IEEE, 2010.

[40] Matson, Johnny L., and Alison M. Kozlowski. "The increasing prevalence of autism spectrum disorders." Research in Autism Spectrum Disorders 5, no. 1 (2011): 418-425.

[41] Mobahi, Hossein, and Karrie G. Karahalios. "HCI applications for aiding children with mental disorders." XRDS: Crossroads, The ACM Magazine for Students 12, no. 
2 (2005): 3-3

[42] Moghadas, M., and H. Moradi. "Analyzing Human-Robot Interaction Using Machine Vision for Autism screening." In 2018 6th RSI International Conference on Robotics and Mechatronics (IcRoM), pp. 572-576. IEEE, 2018.

[43] Moon, Sun Jae, Jinseub Hwang, Harrison Scott Hill, Ryan Kervin, Kirstin Brown Birtwell, John Torous, Christopher J. McDougle, and Jung Won Kim. "Mobile device applications and treatment of autism spectrum disorder: a systematic review and meta-analysis of effectiveness." Archives of Disease in Childhood 105, no. 5 (2020): 458-462.

[44] Ning, Michael, Jena Daniels, Jessey Schwartz, Kaitlyn Dunlap, Peter Washington, Haik Kalantarian, Michael Du, and Dennis P. Wall. "Identification and quantification of gaps in access to autism resources in the United States: an infodemiological study." Journal of Medical Internet Research 21, no. 7 (2019): e13094.

[45] Okumura, Tsukasa, Shuichi Urabe, Katsufumi Inoue, and Michifumi Yoshioka "Cooking activities recognition in egocentric videos using hand shape feature with openpose." In Proceedings of the foint Workshop on Multimedia for Cooking and Eating Activities and Multimedia Assisted Dietary Management, pp. 42-45. 2018.

[46] Picard, Rosalind W. "Affective Computing for HCI." In HCI (1), pp. 829-833. 1999.

[47] Pioggia, Giovanni, Roberta Igliozzi, Marcello Ferro, Arti Ahluwalia, Filippo Muratori, and Danilo De Rossi. "An android for enhancing social skills and emotion recognition in people with autism." IEEE Transactions on Neural Systems and Rehabilitation Engineering 13, no. 4 (2005): 507-515.

[48] Rajagopalan, Shyam, Abhinav Dhall, and Roland Goecke. "Self-stimulatory behaviours in the wild for autism diagnosis." In Proceedings of the IEEE International Conference on Computer Vision Workshops, pp. 755-761. 2013.

[49] Rezazadegan, Fahimeh, Sareh Shirazi, Ben Upcrofit, and Michael Milford. "Action recognition: From static datasets to moving robots." In 2017 IEEE International Conference on Robotics and Automation (ICRA), pp. 3185-3191. IEEE, 2017.

[50] Sapiro, Guillermo, Jordan Hashemi, and Geraldine Dawson. "Computer vision and behavioral phenotyping: an autism case study." Current Opinion in Biomedical Engineering 9 (2019): 14-20.

[51] Simon, Tomas, Hanbyul Joo, Iain Matthews, and Yaser Sheikh. "Hand keypoint detection in single images using multiview bootstrapping." In Proceedings of the IEEE conference on Computer Vision and Pattern Recognition, pp. 1145-1153. 2017

[52] Slovák, Petr, Ran Gilad-Bachrach, and Geraldine Fitzpatrick. "Designing socia and emotional skills training: The challenges and opportunities for technology support." In Proceedings of the 33rd Annual ACM Conference on Human Factors in Computing Systems, pp. 2797-2800. 2015.

[53] Smitha, Kavallur Gopi, and A. Prasad Vinod. "Facial emotion recognition system for autistic children: a feasible study based on FPGA implementation." Medical \& biological engineering \& computing 53, no. 11 (2015): 1221-1229.

[54] Tariq, Qandeel, Scott Lanyon Fleming, Jessey Nicole Schwartz, Kaitlyn Dunlap, Conor Corbin, Peter Washington, Haik Kalantarian, Naila Z. Khan, Gary L. Darmstadt, and Dennis Paul Wall. "Detecting developmental delay and autism through machine learning models using home videos of Bangladeshi children Development and validation study." Fournal of medical Internet research 21, no. 4 (2019): e13822.

[55] Tariq, Qandeel, Jena Daniels, Jessey Nicole Schwartz, Peter Washington, Haik Kalantarian, and Dennis Paul Wall. "Mobile detection of autism through machine learning on home video: A development and prospective validation study." PLoS medicine 15, no. 11 (2018): e1002705.

[56] Voss, Catalin, Jessey Schwartz, Jena Daniels, Aaron Kline, Nick Haber, Peter Washington, Qandeel Tariq et al. "Effect of wearable digital intervention for improving socialization in children with autism spectrum disorder: a randomized clinical trial." JAMA pediatrics 173, no. 5 (2019): 446-454.

[57] Voss, Catalin, Peter Washington, Nick Haber, Aaron Kline, Jena Daniels, Azar Fazel, Titas De et al. "Superpower glass: delivering unobtrusive real-time social cues in wearable systems." In Proceedings of the 2016 ACM International foint Conference on Pervasive and Ubiquitous Computing: Adjunct, pp. 1218-1226. 2016.

[58] Vyas, Kathan, Rui Ma, Behnaz Rezaei, Shuangjun Liu, Michael Neubauer, Thomas Ploetz, Ronald Oberleitner, and Sarah Ostadabbas. "Recognition Of Atypical Behavior In Autism Diagnosis From Video Using Pose Estimation Over Time." In
2019 IEEE 29th International Workshop on Machine Learning for Signal Processing (MLSP), pp. 1-6. IEEE, 2019.

[59] Wall, Dennis Paul, J. Kosmicki, T. F. Deluca, E. Harstad, and Vincent Alfred Fusaro. "Use of machine learning to shorten observation-based screening and diagnosis of autism." Translational psychiatry 2, no. 4 (2012): e100-e100.

[60] Washington, Peter, Emilie Leblanc, Kaitlyn Dunlap, Yordan Penev, Aaron Kline, Kelley Paskov, Min Woo Sun et al. "Precision Telemedicine through Crowdsourced Machine Learning: Testing Variability of Crowd Workers for Video-Based Autism Feature Recognition." Journal of personalized medicine 10, no. 3 (2020): 86.

[61] Washington, Peter, Natalie Park, Parishkrita Srivastava, Catalin Voss, Aaron Kline, Maya Varma, Qandeel Tariq et al. "Data-driven diagnostics and the potential of mobile artificial intelligence for digital therapeutic phenotyping in computational psychiatry." Biological Psychiatry: Cognitive Neuroscience and Neuroimaging (2019).

[62] Washington, Peter, Haik Kalantarian, Qandeel Tariq, Jessey Schwartz, Kaitlyn Dunlap, Brianna Chrisman, Maya Varma et al. "Validity of online screening for autism: crowdsourcing study comparing paid and unpaid diagnostic tasks." fournal of medical Internet research 21, no. 5 (2019): e13668.

[63] Washington, Peter, Emilie Leblanc, Kaitlyn Dunlap, Yordan Penev, Maya Varma, Jae-Yoon Jung, Brianna Chrisman et al. "Selection of trustworthy crowd workers for telemedical diagnosis of pediatric autism spectrum disorder." PSB, 2021.

[64] Washington, Peter, Kelley Marie Paskov, Haik Kalantarian, Nathaniel Stockham, Catalin Voss, Aaron Kline, Ritik Patnaik et al. "Feature selection and dimension reduction of social autism data." In Pac Symp Biocomput, vol. 25, pp. 707-718. 2020 .

[65] Washington, Peter, Catalin Voss, Nick Haber, Serena Tanaka, Jena Daniels, Carl Feinstein, Terry Winograd, and Dennis Wall. "A wearable social interaction aid for children with autism." In Proceedings of the 2016 CHI Conference Extended Abstracts on Human Factors in Computing Systems, pp. 2348-2354. 2016.

[66] Washington, Peter, Catalin Voss, Aaron Kline, Nick Haber, Jena Daniels, Azar Fazel, Titas De, Carl Feinstein, Terry Winograd, and Dennis Wall. "SuperpowerGlass: a wearable aid for the at-home therapy of children with autism." Proceedings of the ACM on interactive, mobile, wearable and ubiquitous technologies 1, no. 3 (2017): 1-22.

[67] Washington, Peter, Serena Yeung, Bethany Percha, Nicholas Tatonetti, Jan Liphardt, and Dennis P. Wall. "Achieving Trustworthy Biomedical Data Solutions."

[68] Wei, Shih-En, Varun Ramakrishna, Takeo Kanade, and Yaser Sheikh. "Convolutional pose machines." In Proceedings of the IEEE conference on Computer Vision and Pattern Recognition, pp. 4724-4732. 2016.

[69] White, Susan W., Lynn Abbott, Andrea Trubanova Wieckowski, Nicole N. Capriola-Hall, Sherin Aly, and Amira Youssef. "Feasibility of automated training for facial emotion expression and recognition in autism." Behavior therapy 49, no. 6 (2018): 881-888.

[70] Wright, Peter, and John McCarthy. "Empathy and experience in HCI." In Proceedings of the SIGCHI conference on human factors in computing systems, pp. 637-646. 2008.

[71] Wu, Shandong, Omar Oreifej, and Mubarak Shah. "Action recognition in videos acquired by a moving camera using motion decomposition of lagrangian particle trajectories." In 2011 International conference on computer vision, pp. 1419-1426. IEEE, 2011.

[72] Ye, Zhefan, Yin Li, Alireza Fathi, Yi Han, Agata Rozga, Gregory D. Abowd, and James M. Rehg. "Detecting eye contact using wearable eye-tracking glasses." In Proceedings of the 2012 ACM conference on ubiquitous computing, pp. 699-704. 2012.

[73] Zhang, Shugang, Zhiqiang Wei, Jie Nie, Lei Huang, Shuang Wang, and Zhen Li. "A review on human activity recognition using vision-based method." fournal of healthcare engineering 2017 (2017)

[74] Zitnick, C. Lawrence, and Piotr Dollár. "Edge boxes: Locating object proposals from edges." In European conference on computer vision, pp. 391-405. Springer, Cham, 2014.

[75] Zunino, Andrea, Pietro Morerio, Andrea Cavallo, Caterina Ansuini, Jessica Podda, Francesca Battaglia, Edvige Veneselli, Cristina Becchio, and Vittorio Murino. "Video gesture analysis for autism spectrum disorder detection." In 2018 24th International Conference on Pattern Recognition (ICPR), pp. 3421-3426. IEEE, 2018. 